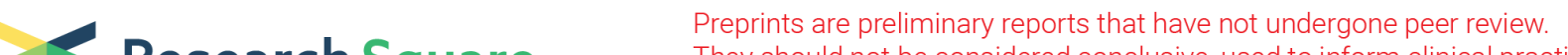 $\begin{array}{ll}\text { Research Square } & \text { They should not be considered conclusive, used to inform clinical practice, } \\ \text { or referenced by the media as validated information. }\end{array}$
}

\section{m(6)A mRNA Methylation Regulates Ferroptosis in HPSCC by Targeting NFE2L2/NRF2}

\section{Jing Ye}

Zhejiang University School of Medicine Sir Run Run Shaw Hospital

\section{Xiaozhen Chen}

Zhejiang University School of Medicine Sir Run Run Shaw Hospital

\section{Bing Liao}

Nanchang University Second Affiliated Hospital

\section{Xiaohua Jiang}

Zhejiang University School of Medicine Sir Run Run Shaw Hospital

\section{Zhihuai Dong}

Zhejiang University School of Medicine Sir Run Run Shaw Hospital

\section{Sunhong $\mathrm{Hu}$}

Zhejiang University School of Medicine Sir Run Run Shaw Hospital

Yuehui Liu

Nanchang University Second Affiliated Hospital

Mang Xiao ( $\square$ joelxm@zju.edu.cn )

Zhejiang University School of Medicine Sir Run Run Shaw Hospital

\section{Research}

Keywords: HPSCC, ALKBH5, m6A, ferroptosis, NFE2L2/NRF2

Posted Date: June 7th, 2021

DOI: https://doi.org/10.21203/rs.3.rs-556028/v1

License: (c) (i) This work is licensed under a Creative Commons Attribution 4.0 International License.

Read Full License 


\section{Abstract \\ Background}

Emerging as the most abundant posttranscriptional internal mRNA modification in eukaryotes, N6methyladenosine $\left(\mathrm{m}^{6} \mathrm{~A}\right)$ modification has gathered tremendous scientific interest in recent years. However, no study addresses the role of $\mathrm{m}^{6} \mathrm{~A}$ modification in ferroptosis. Here, we showed that $\mathrm{m}^{6} \mathrm{~A}$ modifications are decreased in RSL3-induced ferroptosis in hypopharyngeal squamous cell carcinoma (HPSCC). We found that AlkB homolog 5 (ALKBH5), one of the $\mathrm{m}^{6} \mathrm{~A}$ demethylases, is the primary factor involved in aberrant $\mathrm{m}^{6} \mathrm{~A}$ modification.

\section{Methods}

Bioinformatics analysis, sample analysis, cell biological analyses and transcriptome sequencing were performed to evaluate the correlation between $\mathrm{m}^{6} \mathrm{~A}$ modification and ferroptosis as well as molecular mechanism of ALKBH5 function. Transcriptome-wide $\mathrm{m}^{6} \mathrm{~A}$-seq and RIP-seq data and following $\mathrm{m}^{6} \mathrm{~A}$ dot blot, MeRIP-qPCR, RIP-qPCR and dual luciferase reporter assays were mapped to screen and validate the candidate targets of ALKBH5.

\section{Results}

ALKBH5-knockdown impaired ferroptotic cell death in HPSCC. However, overexpression of ALKBH5 has an opposite effect, suggesting that ALKBH5 is a positive regulator of ferroptosis. Mechanistically, ALKBH5-mediated $\mathrm{m}^{6} \mathrm{~A}$ demethylation led to a post-transcriptional inhibition of NFE2L2/NRF2, the central player in the regulation of antioxidant molecules in cells, at two $\mathrm{m}^{6} \mathrm{~A}$ residues in the 3囚-UTR. Therefore, knocking down ALKBH5 subsequently increases the expression levels of NFE2L2/NRF2 and increased cell resistance to ferroptosis. In addition, $\mathrm{m}^{6} \mathrm{~A}$-mediated NFE2L2/NRF2 stabilization relied on the $\mathrm{m}^{6} \mathrm{~A}$ reader IGF2BP2.

\section{Conclusion}

ALKBH5 functions as a tumor suppresser through ferroptosis in HPSCC. ALKBH5 destabilizes NFE2L2/NRF2 expression in HPSCC through an $\mathrm{m}^{6} \mathrm{~A}$-IGF2BP2-dependent mechanism. Together, our work uncovers a critical link between ALKBH5-NFE2L2/NRF2 and ferroptosis, providing insight into the functional importance of the reversible mRNA $\mathrm{m}^{6} \mathrm{~A}$ methylation and its modulators in HPSCC.

\section{Background}


The Head and neck squamous cell carcinoma (HNSCC) are considered as one of the malignities with the most severe impact on quality of life of patients. HNSCC is a heterogeneous group of tumors arising from the mucosal surfaces of the nasal and oral cavity, oropharynx, larynx, and hypopharynx ${ }^{1}$.

Hypopharyngeal squamous cell carcinoma (HPSCC) accounts $3 \%-4 \%$ of HNSCC, however, it is the most invasive cancer among $\mathrm{HNSCC}^{2}$. Although diagnosis and treatment of HPSCC have considerably improved, the survival of recurrent HPSCC can hardly be improved owing to the inadequate effective interventions and precise biomarkers ${ }^{3}$. Therefore, it is quite essential to understand the biological mechanisms of HPSCC malignancy aiming to develop more effective therapeutic strategies.

Triggering apoptotic cell death with anti-cancer drugs is one of the principal approaches for killing cancer cells ${ }^{4}$. However, the effectiveness of apoptosis induction in HNSCC is limited, due to the acquired or intrinsic resistance of cancer cells to apoptosis ${ }^{5-7}$. Ferroptosis is being explored as alternative ways to eradicate apoptosis-resistant cancer cells ${ }^{4}$. It is defined as an iron-catalyzed form of regulated necrosis occurring through excessive peroxidation of polyunsaturated fatty acids (PUFAs) ${ }^{8}$. Recent studies found that the cystine/glutamate antiporter inhibitors erastin and sulfasalazine inhibited head and neck cancer (HNC) cell growth and accumulated lipid reactive oxygen species (lipid ROS), which highlights induction of ferroptotic cell death for killing $\mathrm{HNSCC}^{6}$. However, the molecular regulation for ferroptosis in HPSCC remain unclear.

Emerging as the most abundant posttranscriptional internal mRNA modification in eukaryotes, N6methyladenosine $\left(\mathrm{m}^{6} \mathrm{~A}\right)$ modification has gathered tremendous scientific interest in recent years. The $\mathrm{m}^{6} \mathrm{~A}$ modification in mammalian cells is post-transcriptionally installed by the canonical complex of writers which are comprised of methyltransferase-like 3 (METTL3), methyltransferase-like 14 (METTL14) and Wilms tumor 1 -associated protein (WTAP) ${ }^{9}, 10$. The demethylases can remove the $\mathrm{m}^{6} \mathrm{~A}$ modification which are consist of fat-mass and obesity-associated protein (FTO) and AlkB homolog 5 (ALKBH5) ${ }^{10}$. In addition, $\mathrm{m}^{6} \mathrm{~A}$ modification functions in condition of its recognition by $\mathrm{m}^{6} \mathrm{~A}$ reader proteins. YT521-B homology (YTH) domain-containing family proteins (YTHDF1/2/3) are responsible for mRNA decay ${ }^{11}$. Insulin-like growth factor 2 mRNA binding proteins (IGF2BP1/2/3) responsible for stabilizing mRNA. Accumulating proofs confirmed that the $\mathrm{m}^{6} \mathrm{~A}$ modification regulates multiple biological functions. Besides embryonic development disorders, tumourigenesis, disordered homeostasis and differentiation of immune cells, and nervous system diseases, acquired chemoradio-resistance, inflammatory, autophagy, dominate the $\mathrm{m}^{6} \mathrm{~A}$-related actions in various cancers ${ }^{12-14}$.

Serving as the primary demethylase of $\mathrm{m}^{6} \mathrm{~A}$ modification, ALKBH5 acts as a biological and pharmacological role in human cancers or non-cancers. ALKBH5 plays a dual role in various cancers through regulating kinds of biological processes. The uncovered regulatory mechanisms of ALKBH5dependent $\mathrm{m}^{6} \mathrm{~A}$ modification are implicated with long non-coding RNA, cancer stem cell, autophagy, and hypoxia $^{15}$. ALKBH5 is also characterized as a tumor suppressor in various cancers ${ }^{16-21}$. In current study, we proved that ALKBH5 directly targeted NFE2L2/NRF2 transcripts and mediated their expression in an 
$\mathrm{m}^{6} \mathrm{~A}$-dependent manner. Further study identified that NFE2L2/NRF2 were the targets of IGF2BP2. Upon ALKBH5 knockdown, NFE2L2/NRF2 transcripts with higher $\mathrm{m}^{6} \mathrm{~A}$ levels were captured by IGF2BP2, which resulted in enhanced mRNA stabilization and increased protein expression, thus alleviating ferroptosis. Together, these findings demonstrated the functional importance of the $\mathrm{m}^{6} \mathrm{~A}$ methylation machinery in ferroptosis regulation, which expands our understanding of such interplay that is essential for development of therapeutic strategies in HPSCC.

\section{Methods}

\section{Patient specimens}

HPSCC patients from individual medical centers who underwent surgery and regular medical surveillance between 2012 and 2020 were used in this study. Paraffin-embedded surgical specimen was stored permanently in Sir Run Run Shaw hospital pathology department.

Global $\mathrm{m}^{6} \mathrm{~A}$ measurement

The global $\mathrm{m}^{6} \mathrm{~A}$ levels in mRNA were measured with EpiQuik $\mathrm{m}^{6} \mathrm{~A}$ RNA Methylation Quantification Kit (Colorimetric) (Epigentek, Farmingdale, NY) following the manufacturer's protocol.

$\mathrm{m}^{6} \mathrm{~A}$-sequencing ( $\mathrm{m}^{6} \mathrm{~A}$-seq, MeRIP-seq) and RNA-sequencing (RNA-seq)

Total RNA was extracted using Trizol reagent (Invitrogen, CA, USA) following the manufacturer's procedure. The total RNA quality and quantity were analysis of Bioanalyzer 2100 and RNA 6000 Nano LabChip Kit (Agilent, CA, USA) with RIN number > 7.0. Approximately more than 200 ug of total RNA was subjected to isolate Poly (A) mRNA with poly-T oligo attached magnetic beads (Invitrogen). Following purification, the poly(A) mRNA fractions is fragmented into 100-nt-long oligonucleotides using divalent cations under elevated temperature. Then the cleaved RNA fragments were subjected to incubated for $2 \mathrm{~h}$ at $4^{\circ} \mathrm{C}$ with $\mathrm{m}^{6} \mathrm{~A}$-specific antibody (No. 202003, Synaptic Systems, Germany) in IP buffer (50 mM Tris$\mathrm{HCl}, 750 \mathrm{mM} \mathrm{NaCl}$ and $0.5 \%$ Igepal CA-630) supplemented with BSA $(0.5 \mu \mathrm{g} \mu \mathrm{l}-1)$. The mixture was then incubated with protein-A beads and eluted with elution buffer $\left(1 \times \mathrm{IP}\right.$ buffer and $\left.6.7 \mathrm{mM} \mathrm{m}^{6} \mathrm{~A}\right)$. Eluted RNA was precipitated by $75 \%$ ethanol. Eluted $\mathrm{m}^{6} \mathrm{~A}$-containing fragments $(\mathrm{IP})$ and untreated input control fragments are converted to final CDNA library in accordance with a strand-specific library preparation by dUTP method. The average insert size for the paired-end libraries was $\sim 100 \pm 50 \mathrm{bp}$. And then we performed the paired-end $2 \times 150 \mathrm{bp}$ sequencing on an Illumina Novaseq ${ }^{\text {TM }} 6000$ platform at the LC-BIO Biotech Itd (Hangzhou, China) following the vendor's recommended protocol.

Anti-m ${ }^{6} \mathrm{~A}$ immunoprecipitation and RNA immunoprecipitation(RIP)

Cells were washed twice with PBS, collected and then the pellet was resuspended in IP lysis buffer $(150 \mathrm{mM} \mathrm{KCl}, 25 \mathrm{mM}$ Tris (pH 7.4), $5 \mathrm{mM}$ EDTA, $0.5 \mathrm{mM}$ DTT, $0.5 \% \mathrm{NP} 40,1 \times$ protease inhibitor, $1 \mathrm{U} / \mu \mathrm{l}$ RNase inhibitor). The lysate was harvested by centrifugation at $12000 \mathrm{~g}$ for $10 \mathrm{~min}$ after incubation for 
30 min. Antibodies and $40 \mu$ l of protein $\mathrm{G}$ beads (Invitrogen, USA) were added into the lysate followed by incubation overnight at $4^{\circ} \mathrm{C}$. After washed three times with wash buffer $(150 \mathrm{mM} \mathrm{KCl}, 25 \mathrm{mM}$ Tris $(\mathrm{pH} 7.4)$, 5mM EDTA, 0.5mM DTT, 0.5\% NP40), co-precipitated RNAs were extracted by Trizol reagent, ethanolprecipitated with glycogen (Invitrogen, USA). The enrichment of RNAs was normalized to lgG.

\section{Luciferase assay}

The fragments of NRF2-3囚-UTR containing the wild-type m6A motifs as well as mutant $\mathrm{m}^{6} \mathrm{~A}$ motifs $\left(\mathrm{m}^{6} \mathrm{~A}\right.$ was replaced by $\mathrm{C}$ ) were synthesized at Gene Chemistry(Shanghai, China). The firefly luciferase and Renilla luciferase activities in each well were calculated by a dual-luciferase reporter assay system. The ratios between the 3囚-UTR reporter of NRF2 and Renilla control were determined $48 \mathrm{~h}$ after siRNA treatment. The relative luciferase activity was further normalized to that in cells transfected with the firefly luciferase vector control under the same treating conditions. Firefly luciferase activity and Renilla luciferase activity were measured using FLUOstar Omega (BMG LABTECH, Offenburg, Germany). The results were shown in the form of relative firefly luciferase activity normalized to Renilla luciferase activity. All the experiments were repeated for three times, and three replicates were conducted for each group.

Mouse treatment and tumor biology studies

HPSCC FaDu or Detroit 562 cell lines $\left(1 \times 10^{6}\right.$ cells) stably expressing vector control and construct lentivirus were injected subcutaneously into the right flank of nude mice. Tumor sizes and mice weight in all groups were measured every 3 days for 1-6 weeks. For NRF2 inhibitors treatment, male nude mice at 4 weeks of age were injected subcutaneously into the right flank with Detroit 562 and FaDu cell lines ( $1 \times 10^{6}$ cells). After 12 days, mice were divided into control and NRF2 inhibitors groups ( $n=5$ per group). NRF2 inhibitors concentration: $30 \mathrm{mg} / \mathrm{kg}$ intraperitoneal injection.

All mice were maintained in standard cages in a light and temperature-controlled room and were given standard chow and water. All animal studies were carried out in accordance with Institute of Laboratory Animal Resources guidelines and approved by the University Committee on the Use and Care of Animals at the Zhejiang University.

Statistics methods

Bioinformatic analysis including GO, KEGG, GSEA,circos plot analysis were performed using the OmicStudio tools at https://www.omicstudio.cn/tool. TCGA HNSCC data analysis were performed using GEPIA $^{36}$. NFE2L2/NRF2 functional association data including the protein and genetic interactions, pathways, co-expression, co-localization is summarized by GeneMANIA (http://genemania.org). All statistical analysis was carried out using GraphPad Prism version 7 (GraphPad Software, CA) for Windows. Statistical significance was assessed by unpaired two tailed Student's-tests, analysis of variance tests (ANOVA) or Spearman rank correlation. The data are expressed as mean $\pm S D$. ${ }^{*} P<0.05$; ${ }^{* *} P<0.01 ; * \star * P<0.001$. Each experiment was repeated independently at least three times. 


\section{Results}

\section{Sensitivity to ferroptosis was associated with $\mathrm{m}^{6} \mathrm{~A}$ modification level in HNSCC.}

RSL3 is an activator of ferroptosis, which inhibits the activity of GPX4 by covalent bonding with GPX4 and leads to lipid peroxides accumulation ${ }^{22}$. Firstly, we performed different HNSCC cell lines exposed to RSL3 with various concentrations for 24 hours. AlamarBlueassay and LDH release assay showed that sensitivity to RSL3 treatment differed considerably between the various HNSCC cell lines. RSL3 reduced

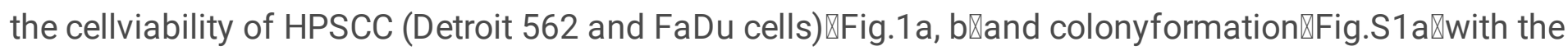
sensitivity greatly decreased inOSCC (SCC9 and SCC25) cells.Cell death induced in HPSCC cells by RSL3

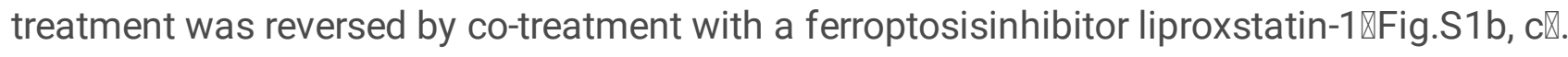

Meanwhile, RSL3 induced cellular lipid peroxidation (lipid ROS) levels to varying degrees among HNSCC cell lines.Changes in cellular lipid ROS measured by BODIPY C11 after RSL3

treatment showed a similar trend in these cell lines $\mathbb{F} F i g .1 \mathrm{C} \mathbb{R}$.RSL3 reduced the expression of GPX4 protein in a dose-dependent manner in Detroit 562 HPSCC cell, but not in SCC25 cells $\varangle$ Fig. $1 \mathrm{~d} \rrbracket$.

To explore the correlation between $\mathrm{m}^{6} \mathrm{~A}$ modificationswith ferroptosis in $\mathrm{HNSCC}, \mathrm{m}^{6} \mathrm{~A}$ dot blot experiments and $\mathrm{m}^{6} \mathrm{~A}$ RNA Methylation Quantification were performed. The results showed that the $\mathrm{m}^{6} \mathrm{~A}$ modification level was significantly decreased in HPSCC cells which showed higher sensitivity to ferroptosis) $\nabla$ Fig.1e, $\mathrm{f} \otimes$.Furthermore, qRT-PCR analysis was performed to compare theregulated- $\mathrm{m}^{6} \mathrm{~A}$ gene expression profiles in HPSCC cells, SCC9 and SCC25. In line with $\mathrm{m}^{6} \mathrm{~A}$ modification level lower in HPSCC, we observed that there was a most significant higher ALKBH5 expression in HPSCC (Fig.1g). It implies that dysregulation of ALKBH5 may be involved in ferroptosis of HPSCC.

\section{Inhibition of ALKBH5 expression decrease sensitivity toferroptosis in HPSCC.}

HPSCC cells were treated with the lentivirus encoding a short hairpin RNA specific for ALKBH5.The transfection efficiency was validated by qRT-PCR and western blotting (Fig. S2a, b). A noticeable increase in $\mathrm{m}^{6} \mathrm{~A}$ levels was observed in polyadenylated RNAs (poly $(\mathrm{A}) \mathrm{RNAs}$ ) of the ALKBH5-knockdown cells, compared to the wild-type (WT)HPSCC cells, asmeasured by $\mathrm{m}^{6} \mathrm{~A}$ dot blot (Fig. 2a) and EpiQuik ${ }^{\mathrm{TM}} \mathrm{m} 6 \mathrm{~A}$ quantificationassay (Fig. S2c).

To elucidate whether ALKBH5 plays a role in HPSCC ferroptosis, we performed western blotting to compare the novel ferroptosis-related gene expression of ALKBH5-knockdown Detroit 562 cells and 
control cells. Knocking down ALKBH5 remarkedly increased the expression of GPX4, HO-1 and NRF2 protein in shALKBH5 cells, but not in control cells, along with the decreased expression of TFRCand Keap1 protein(Fig. 2b). Immunofluorescence staining showed higher positivity of GPX4 in shALKBH5 cells with RSL3 treatment, compared with control cells (Fig. 2c). Inhibition of the ALKBH5 gene significantly reversed RSL3-induced cell death and cellular lipid ROS levels (Fig. 2d-f). Along with that, using electron microscopy we observed much more shrunken mitochondria in shCON cells than shALKBH5 FaDu cells with RSL3 treatment (Fig. 2g). These data suggested that ALKBH5 positively regulates RSL3-induced ferroptosis.

\section{The overexpression of ALKBH5 sensitized HPSCC cells to RSL3 treatment in vitro and vivo}

We next examined the effect of ALKBH5 overexpression on cell growth and viability of HPSCC cells. The transfection efficiency was validated by qRT-PCR and western blotting(Fig. S2d). Inhibition of NRF2 and GPX4 could be induced by the ALKBH5 transfection. When the ALKBH5 gene was overexpressed by plasmid transfection, NRF2 and GPX4 expression decreased in HPSCC with or without RSL3 treatment (Fig.3a). Meanwhile, ALKBH5 overexpression inhibitsthe growth and cell viability of HPSCC cells (Fig.3bd). The cell death was enhanced by a combination of RSL3 with ALKBH5 plasmid transfection (Fig.3b-d). To clarify the effects of ALKBH5 on HPSCCin vivo, ALKBH5-overexpressing and negative control FaDu cells were implanted into BALB/c mice via subcutaneous injection.RSL3 treatment reduced HPSCC tumor growth and tumor weight in xenograft mouse models (Fig. 3e, f). In addition, overexpression of ALKBH5 significantly enhanced the RSL3-inducded tumor suppression. The data strongly suggests that ALKBH5 may be a tumor-suppress gene and mediate ferroptosis in HPSCC.

\section{MeRIP-seq combined with RNA-seq identified potential targets of ALKBH5.}

To identify potential mRNA targets of ALKBH5 whose $\mathrm{m}^{6} \mathrm{~A}$ levels were decreased by ALKBH5 in HPSCC cells, ALKBH5-knockdown and control FaDu cells were selected for transcriptome-wide $\mathrm{m}^{6} \mathrm{~A}$-sequencing ( $\mathrm{m}^{6} \mathrm{~A}$-seq, MeRIP-seq) and RNA-sequencing (RNA-seq) assays. Principal component analysis (PCA) showed that two repeats (shCON: shCON_1 and shCON_2; shALKBH5: shK5_1 and shK5_2) of each sample clustered together, suggesting good repeatability among the two replicates of each group (Fig. $\mathrm{S} 3 \mathrm{a})$. Consisted with the previous research, the most common $\mathrm{m}^{6} \mathrm{~A}$ motif "GGAC" is significantly enriched in the $\mathrm{m}^{6} \mathrm{~A}$ peaks (Fig. 4a).RNA-seq uncovered 548 down-regulated and 564 up-regulated transcripts ( $\mathrm{p}$ 0.05) upon ALKBH5 overexpression(Fig. S3b). Peaks were located in protein-coding transcripts and enriched in the $5^{\prime} \mathrm{UTR}$ and $3^{\prime} \mathrm{UTR}$, especially near stop codons, which was coincidence with the $\mathrm{m}^{6} \mathrm{~A}$ distribution(Fig. 4b, c). 
We next compared the genes with altered- $\mathrm{m}^{6} \mathrm{~A}$ modifications between the control and ALKBH5knockdown cells. The analysis of $\mathrm{m}^{6} \mathrm{~A}$-seq revealed a global hypermethylation of $\mathrm{m}^{6} \mathrm{~A}$ in the transcription level after knockdown of ALKBH5 in FaDu cells (Fig. 4d). Considering the role of ALKBH5 in the $\mathrm{m}^{6} \mathrm{~A}$ methyltransferase complex, mRNA transcripts carrying hypermethylated

$\mathrm{m}^{6} \mathrm{~A}$ peaks in FaDu ALKBH5 knockdown cells were likely potential targets. Through analysis of the RNAseq data, we identified 967 hyper-methylated $m^{6} \mathrm{~A}$ genes whose mRNA transcripts were down-regulated ( $p$ $<0.05$, Hyper-down) and 49 hyper-methylated $\mathrm{m}^{6} \mathrm{~A}$ genes whose mRNA transcripts were up-regulated $(\mathrm{p}<$ 0.05, Hyper-up) in ALKBH5-knockdown cells, compared with shCON cells (Fig. 4e). GO enrichment and KEGG pathway analysis revealed that these hyper-methylated $m^{6}$ Agene transcripts $(p<0.05)$ were predominantly enriched in "protein and lipid binding", "lipid metabolic process", "membrane", "endocytosis" and "ferroptosis" (Fig. S3c). Moreover, GSEA showed that these genes are involved in response_to_oxidized_phospholipids, KEGG_oxidative_phosphorylation, missiaglia _regulated _by_methylation_up (Fig. S3d).It is consisting with the acknowledge that lipid peroxidation of long chain fatty acid and oxidative phosphorylation were correlated with cell membrane oxidative damage in ferroptosis process ${ }^{23-26} .182$ genes involved in ferroptosis and lipid oxidation andoxidoreductase activity were selected for further validation. Six genes STEAP3, AIFM2, NFE2L2/NRF2, FH(Fumarate hydratase), ZNRF2, PEBP1 whose alterations suppress ferroptosis were finally selected as candidate targets of ALKBH5(Fig. 4f).

Further qRT-PCR analyses showed the mRNA levels in NFE2L2/NRF2 genes were dramatically increased in ALKBH5-knockdown FaDu cells (Fig. 4g). The Integrative Genomics View (IGV) data peak revealed $\mathrm{m}^{6} \mathrm{~A}$ peak enrichment in the 5'-UTR of NFE2L2/NRF2 mRNA that was diminished upon ALKBH5 knockdown (Fig. 4h, i).Consistent with our results, previously reported $\mathrm{m}^{6} \mathrm{~A}$, RIP and CLIP data from the $\mathrm{m}^{6} \mathrm{~A} 2 \mathrm{Target}$ Database (http://m6a2target.canceromics.org/\#/search/TFR) identified NFE2L2/NRF2 mRNA as a potential target of ALKBH5 (GSE87515).

NFE2L2/NRF2 plays the crucial role in the regulation of cellular antioxidant molecules. It controls cellular antioxidant systems in cancer cells, playing a key role in protecting against intracellular and environmental stress ${ }^{27,28}$. NFE2L2/NRF2 is constantly degraded by Keap1 and is activated by the inhibition of Keap $1^{29,30}$. Ferroptosis could be a result of aberrant NRF2 signaling ${ }^{31}$. Inhibition of NFE2L2/NRF2 and the AREs HO-1, FTH1, and NQO1 in HNCcells has been shown to significantly enhance the antitumor activity of ferroptosis inducers ${ }^{32}$, 33. Furthermore, accumulation of the NFE2L2/NRF2-ARE proteins during ferroptosis reduced the sensitivity of head and neck cells to the GPX4 inhibitors RSL3 and ML-162 ${ }^{34}$. Taken together, NFE2L2/NRF2 may be the direct downstream target of ALKBH5.

\section{ALKBH5 abolishes NFE2L2/NRF2mRNA levels and stability depending on its $\mathrm{m}^{6} \mathrm{~A}$ methyltransferase activity.}


MeRIP-qPCR assays with specific primers aiming at potential $\mathrm{m}^{6} \mathrm{~A}$ sites was then applied to confirm the ALKBH5-mediated $\mathrm{m}^{6} \mathrm{~A}$ demethylation of NFE2L2/NRF2mRNAin 3' UTR. When compared to the IgG group (pulldown control), an enrichment of NFE2L2/NRF2 mRNA in 3' UTR was obtained by the reaction to $\mathrm{m}^{6} \mathrm{~A}$ specific antibody (Fig. 5a).Western blotting and qRT-PCR was then applied to confirm NFE2L2/NRF2expression was upregulated in ALKBH5-knockdown FaDu cells(Fig. S4a).

To elucidate whether the ALKBH5 demethyltransferase function decreased NFE2L2/NRF2 expression, ALKBH5 mutant (ALKBH5-mut, NM_017758, H204A) recombination plasmids was constructed. Then we measured $\mathrm{m}^{6} \mathrm{~A}$ levels of NFE2L2/NRF2 after transfection of control, ALKBH5-WT and ALKBH5-mut plasmids, The MeRIP-qPCR data showed that overexpression of ALKBH5-WT, but not ALKBH5-mut, dramatically led to a decreased $\mathrm{m}^{6} \mathrm{~A}$ levelsof NFE2L2/NRF2 in Detroit562 cells (Fig. 5b).In addition, overexpression of ALKBH5, but not ALKBH5-mut dramatically decreased NFE2L2/NRF2expression (Fig. S4b).

Thereafter, to address the effect of $\mathrm{m}^{6} \mathrm{~A}$ modification on NFE2L2/NRF2 gene,we constructed both wildtype and mutant NFE2L2/NRF2 luciferase reporter plasmids, whichN6-methylated adenosine (A)was replaced with $\mathrm{C}$ (cytosine) ofNFE2L2/NRF2 mRNA that have intact $\mathrm{m}^{6} \mathrm{~A}$ sites (Fig. 4i, Fig. 5c, Fig. S4c).As expected, compared with shCON, ALKBH5-knockdown substantially increased luciferase activity of the individual reporter (Firefly/Renilla) constructs containing wild-type NFE2L2/NRF2 3'UTR. However, ALKBH5-knockdown had no such significant effect on the NFE2L2/NRF2 -Mut luciferase reporter plasmid in Detroit 562 and FaDu cells (Fig. 5d). Furthermore, overexpression of ALKBH5, but not ALKBH5 mutant, significantly reduced luciferase activity of individual reporter (Firefly/Renilla) constructs containing wildtype NFE2L2/NRF23' UTR (Fig. 5e). Overexpression of ALKBH5 and ALKBH5-mut had no such significant effect on NFE2L2/NRF23' UTR luciferase reporterplasmids (Fig. 5e).

The $\mathrm{m}^{6} \mathrm{~A}$-modification is interpreted by its readers, such as YTH domain-containing proteins, to regulate mRNA fate; insulin-like growth factor 2 mRNA-binding proteins (IGF2BPs; including IGF2BP1/2/3), to promote the stability and storage of their target mRNAs in an $\mathrm{m}^{6} \mathrm{~A}$-dependent manner under normal and stress conditions and therefore affect gene expression output ${ }^{35}$. To analyze the effect of $\mathrm{m}^{6} \mathrm{~A}$ modification on the stability of ALKBH5 target transcripts, we conducted RNA stability assays. The RNA stability curves showed that knockdown of ALKBH5 prolong the half-life of NFE2L2/NRF2 mRNA in HPSCC cells (Fig. 5f). As YTHDF2, YTHDF3 and YTHDC2 are responsible for mRNA decay, they were hardly the potential effectors participating in the current process.Nevertheless, qRT-PCR data showed that knockdown of IGF2BP2, but not IGF2BP1/3 significantly reduced the mRNA level of NFE2L2/NRF2 in HPSCC cells(Fig. S4d,e). Furthermore, the half-life of NFE2L2/NRF2 mRNA was significantly decreased when IGF2BP1 was impaired (Fig. S4f). Notably, NFE2L2/NRF2 expression was positively correlated with the IGF2BP2 level in the TCGA HNSCC dataset (Fig. S4g) ${ }^{36}$. RIP- assay showed that IGF2BP2 directly bound to the 3' UTR of NFE2L2/NRF2 mRNA (Fig. 5g). RIP-PCR indicated that knockdown of ALKBH5 significantly enhanced the binding efficiency of IGF2BP2 to the 3' UTR of NFE2L2/NRF2 mRNA(Fig. 5h).In 


\section{NFE2L2/NRF2 is functionally essential target gene of ALKBH5 in HPSCC.}

We next performed rescue-experiments to illustrate whether NFE2L2/NRF2 participated in the biological function of ALKBH5 in HPSCC. Detroit 562 control and ALKBH5-knockdown cells were transfected with control and siNFE2L2/NRF2 (siNRF2), while FaDu control and ALKBH5-knockdown cells were transfected with control and NFE2L2/NRF2-WT plasmids. The transfection efficiency of were confirmedby qRT-PCR and Western blot analysis (Fig. S5a, b). NFE2L2/NRF2-overexpression downregulated protein levels of GPX4 and FTH1 of ALKBH5-knockdowncells (Fig. S5c). Cell viability assay, LDH release assay and Colonyformation assays indicated that silencing of NFE2L2/NRF2 impairedcell growth and viability of ALKBH5-knockdown cells (Fig. 6a-c). Silencing of NFE2L2/NRF2 also significantly increased cellular lipid ROS levelsof ALKBH5-knockdown cells (Fig. 6d). NFE2L2/NRF2 overexpression also led to significantly restored cell growth and viability of ALKBH5-overexpression cells (Fig. S5d, e). These results strongly indicated that NFE2L2/NRF2 is a critical target gene of ALKBH5 in HPSCC cells. Inhibition of GPX4 renders HNC cells susceptible to ferroptosis, while activation of the Nrf2-ARE system caused the resistance to GPX4 inhibition 22,34 . Both the light chain and heavy chain of ferritin (FTL/FTH1), stores iron in ferritin, as well as ferroportin (SLC40A1), which is responsible for iron efflux out of the cell, are controlled by NRF2 ${ }^{37,38}$. Furthermore, western blot analysis showed that NFE2L2/NRF2 silencing restored the protein levels of GPX4 and FTH1 of ALKBH5-knockdown cells (Fig. 6e). Analysis of TCGA HNSCC datasets also revealed high GPX4 levels positively correlated with NFE2L2/NRF2 expression (Fig. S5f) ${ }^{36}$.

So far, we have proved that ALKBH5 may function as a tumor suppresser through ferroptosis by abolishing NFE2L2/NRF2 mRNA stability and expression in HPSCC cells. Several small molecular NRF2 inhibitors have been developed as anti-tumor drug candidates ${ }^{39}$, 40 . We treated the mice baring shALKBH5 and control xenograft tumor with one NRF2 inhibitor-ML385 ${ }^{41,42}$. ML385 is efficient to inhibit tumor growth and tumor weight in nude mice bearing shALKBH5 cells (Fig. 6f-h). We next performed IHC analyses of HPSCC and paracancerous tissues from patients, to further explore the correlation between expression of ALKBH5 and NFE2L2/NRF2 in HPSCC tissues. As expected, ALKBH5 negatively interrelated with NFE2L2/NRF2 expression (Fig. 6i, j). Taken together, the data show that NFE2L2/NRF2 mediates the ferroptosis regulation of ALKBH5 in HPSCC cells.

\section{Discussion}

The $\mathrm{m}^{6} \mathrm{~A}$ modification exerts direct control over the RNA metabolism including mRNA processing, mRNA exporting, translation initiation, mRNA stability and the biogenesis of long-non-coding RNA (LncRNA), thereby can influence various aspects of cell function. Accumulating evidence demonstrates that $\mathrm{m}^{6} \mathrm{~A}$ 
modulates gene expression, thereby regulating cellular processes ranging from differentiation, apoptosis, therapeutic resistance, and immune response ${ }^{43,44}$.

ALKBH5-directed $\mathrm{m}^{6} \mathrm{~A}$ demethylation is involved in splicing and the production of longer $3^{\prime}$-UTR mRNAs. Regulatory mechanisms of ALKBH5 including $\mathrm{m}^{6} \mathrm{~A}$-dependent modification implicated with long noncoding RNA, cancer stem cell, autophagy, and hypoxia.In epithelial ovarian cancer (EOC), up-regulated ALKBH5 impairs the autophagy and facilitates cell proliferation and invasion ${ }^{45}$.METTL3 relieved autophagic flux in hypoxia/reoxygenation treated cardiomyocytes, however ALKBH5 could reverse this effect $^{16}$. Here our work firstly uncovered a critical link between ALKBH5-NFE2L2/NRF2 and ferroptosis in HPSCC (Fig.7). Knockdown ALKBH5 dramatically suppress the cell response to RSL3-induced ferroptosis. Overexpression ALKBH5 remarkably enhanced the ferroptotic cell death and lipidperoxidation.

Ferroptosis is an iron-dependent, lipid peroxidation-driven cell death cascade that shows great potentials during the cancer therapy ${ }^{38,46}$. Several small molecules have been identified to be functioning in ferroptosis initiation and ferroptosis sensitivity in different cancers ${ }^{38,46}$. A clear understanding of ferroptosis sensitivity in cancers will benefit the clinical practice in applying ferroptosis to cancer therapy. Importantly we found ALKBH5 expression enhanced sensitivity to ferroptosis in HPSCC in $\mathrm{m}^{6} \mathrm{~A}$-dependent manner.

By RNA-seq and $\mathrm{m}^{6} \mathrm{~A}$-seq analysis, we identified NFE2L2/NRF2 as a downstream target of ALKBH5mediated $\mathrm{m}^{6} \mathrm{~A}$ modification, which was further convinced by the MeRIP-qPCR analysis, luciferase and qRT-PCR assays in control and ALKBH5-knockdown FaDu cells. Tremendous studies highlightedthatNFE2L2/NRF2 is a critical mitigator of both lipid peroxidation and ferroptosis, established NRF2 target genes that mitigate these pathways, as well as the relevance of the NRF2-lipid peroxidationferroptosis axis in disease ${ }^{38}$. Keap1-NRF2 antioxidant system is involved in the RSL3-induced resistance mechanism ${ }^{47,48}$. NFE2L2/NRF2 inhibition or p62 silencing sensitized chemoresistant HNC cells to RSL3. Activation of the NRF2-ARE pathway contributed to the resistance of HNC cells to GPX4 inhibition, and that inhibition of this pathway reversed the resistance to ferroptosis in HNC cells.As discussed in detail in prior studies, GPX4 is an established NFE2L2/NRF2 transcriptional target, is an integral anti-ferroptotic reducer of lipid peroxides $31,38,49$. NFE2L2/NRF2 functional association data including the protein and genetic interactions, pathways, co-expression, co-localization is summarized byGeneMANIA (http://genemania.org). Therefore, inthe current study we did not go into details to explore the potential mechanisms by which NFE2L2/NRF2 regulates the GPX4-dependent lipid peroxidation.

There is also an essential link between NFE2L2/NRF2 function and iron homeostasis ${ }^{38}$. Our previous work revealed that YTHDF1 is closely correlated with iron metabolism and tumor progression in HPSCC. Mechanistically, YTHDF1 enhanced transferrin receptors (TFRC) expression through an $\mathrm{m}^{6} \mathrm{~A}$-dependent mechanism ${ }^{50}$. To determine whether YTHDF1 recognized the $\mathrm{m}^{6} \mathrm{~A}$ modification on NFE2L2/NRF2, we reanalyzed the sequence data, in which YTHDF1-knockdown and control FaDu cells were subjected. The $\mathrm{m}^{6} \mathrm{~A}$-seqdata indicated $\mathrm{m}^{6} \mathrm{~A}$ peaks in the mRNA and gene expression change. but MeRIP-qPCR failed to 
validate this finding (data not shown). Then we speculated that ALKBH5 stabilizes NFE2L2/NRF2

expression through RNA stabilization. The defined $\mathrm{m}^{6} \mathrm{~A}$ site is located around the $\mathrm{m}^{6} \mathrm{~A}$ enrichment regions in the 5 ' UTR. We then observed a significantly augmented NFE2L2/NRF2 mRNA stability in HPSCC cells upon the knockdown of IGF2BP2. RIP assay confirmed the binding between IGF2BP2 and NFE2L2/NRF2 mRNA. Functional rescue experiments were additionally performed. Silencing of NFE2L2/NRF2 impaired cell growth and upregulated cellular lipid ROS levels of ALKBH5-knockdown cells (Fig. 6a-d).

In addition, NRF2 inhibitor has anti-cancer effect on ALKBH5-knockdown xenograft tumor (Fig. 6f-h). As ALKBH5 functions as a tumor suppresser through ferroptosis in HPSCC, we speculated that targeting NRF2 may be more efficient in low-expression ALKBH5 tumor via inhibiting the ferroptosis-resistance. These hypotheses need to be validated in vivo experiments in future.

The prognostic value role of ALKBH5 in HPSCC progress may not be fully unveiled in present study. Actually, the role of ALKBH5 in human cancers was still controversial. It had been found to play an oncogenic or tumor suppressive role in different kinds of cancers ${ }^{51}$. In our study,ALKBH5 was characterized as a critical regulator linking ferroptosis and $\mathrm{m}^{6} \mathrm{~A}$ modification. Therefore, at least we proved ALKBH5 could be functioning in ferroptosis sensitivity in HPSCC. Inducing ferroptosis would be more efficient in cancer therapy among high-expressed ALBH5 HPSCC.

Taken together, ALKBH5 leads to impaired NFE2L2/NRF2 mRNA stability and decreased expression in HPSCC through an $\mathrm{m}^{6} \mathrm{~A}-\mathrm{IGF} 2 \mathrm{BP} 2$-dependent mechanism. Our work uncovers a critical link between ALKBH5-NFE2L2/NRF2 and ferroptosis, providing insight into the functional importance of the reversible mRNA $\mathrm{m}^{6} \mathrm{~A}$ methylation and its modulators in HPSCC.

\section{Conclusions}

Here, for the first time, we found that $\mathrm{m}^{6} \mathrm{~A}$ modification is closely correlated with ferroptosis pathway in HNSCC cells. NFE2L2/NRF2 mRNA were found to be regulated by $\mathrm{m}^{6} \mathrm{~A}$-modification. The ALKBH5NFE2L2/NRF2-IGF2BP2 axislikely plays a critical role in the ferroptosis of HPSCC. Ferroptosis as a anticancer therapy may bepromising for treating HPSCC patients withhigh ALKBH5 expression.

\section{Abbreviations}

GPX4:Glutathione peroxidase

HNSCC: Head and neck squamous cell carcinoma

HPSCC: Hypopharyngeal carcinomas

FTH1: Ferritin heavy chain 1

GPX4: Glutathione peroxidase 4 
IGV: Integrative Genomics View

IHC: Immunohistochemical staining

KEGG: Kyoto Encyclopedia of Genes and Genomes

Lipro-1:Liproxstatin-1

NRF2: The transcription factor nuclear factor erythroid 2-related factor 2

TFRC: Transferrin receptors

ROS: Reactive oxygen species

\section{Declarations}

\section{Ethics approval and consent to participate.}

According to the protocol approved by the Ethical Review Committee of the Sir Run Run Shaw Hospital, College of Medicine, Zhejiang University. Patient tissue samples were obtained with informed consent. The in vivo assay using nude mice was approved by the Ethics Review Committee of Zhejiang University college of Medicine.

\section{Availability of data and materials}

The original patient data from Sir run run Shaw hospital biological specimen Bank are accessible on request for non-commercial purposes.

\section{Consent for publication}

All authors have agreed to publish this manuscript.

\section{Competing interests}

The authors declare that they have no competing interests.

\section{Funding}

This work was supported by the Natural Science Foundation of Zhejiang Province (No. LY21H160031), National Natural Science Foundation of China (No. 62071415, No.81903160), Medical Health Science 
and Technology Project of Zhejiang Provincial Health Commission Grants (No. 2019336033 and No. 2020367813).

\section{Authors' contributions}

Conceptualization: Mang Xiao

Datacuration: Mang Xiao

Formal analysis: Jing Ye, Bing Liao, Xiaozhen Chen

Funding acquisition: Yuehui Liu, Mang Xiao

Investigation: Xiaohua Jiang, Bing Liao

Methodology: Jing Ye, Xiaozhen Chen

Project administration: Jing Ye, Xiaozhen Chen,

Resources: Yuehui Liu, Sunhong Hu, Bing Liao

Software: Zhihuai Dong,

Supervision: Yuehui Liu, Mang Xiao

Validation: Mang Xiao

Visualization: Yuehui Liu, Jing Ye

Roles/Writing - original draft: Jing Ye, MangXiao

Writing - review \& editing: Yuehui Liu, Mang Xiao

\section{Acknowledgements}

Not applicable.

\section{References}

1. Peltanova B, Raudenska M, Masarik M. Effect of tumor microenvironment on pathogenesis of the head and neck squamous cell carcinoma: a systematic review. Mol Cancer. 2019;18:63.

2. Yamashita Y, Ikegami T, Suzuki M, et al. Hypopharyngeal cancer risk in Japanese: Genetic polymorphisms related to the metabolism of alcohol- and tobacco-associated carcinogens. J Cancer Res Ther. 2019;15:556-563. 
3. Joo YH, Lee YS, Cho KJ, et al. Characteristics and prognostic implications of high-risk HPVassociated hypopharyngeal cancers. Plos One. 2013;8:e78718.

4. Hassannia B, Vandenabeele P, Vanden BT. Targeting Ferroptosis to Iron Out Cancer. Cancer Cell. 2019;35:830-849.

5. Liu K, Hu H, Jiang $\mathrm{H}$, et al. Upregulation of secreted phosphoprotein 1 affects malignant progression, prognosis, and resistance to cetuximab via the KRAS/MEK pathway in head and neck cancer. Mol Carcinog. 2020;59:1147-1158.

6. Roh JL, Kim EH, Jang HJ, Park JY, Shin D. Induction of ferroptotic cell death for overcoming cisplatin resistance of head and neck cancer. Cancer Lett. 2016;381:96-103.

7. Zhao M, Mydlarz WK, Zhou S, Califano J. Head and neck cancer cell lines are resistant to mitochondrial-depolarization-induced apoptosis. ORL J Otorhinolaryngol Relat Spec. 2008;70:257263.

8. Dixon SJ, Lemberg KM, Lamprecht MR, et al. Ferroptosis: An Iron-Dependent Form of Nonapoptotic Cell Death. Cell. 2012;149:1060-1072.

9. Meyer KD, Jaffrey SR. Rethinking m6 A Readers, Writers, and Erasers. Annu Rev Cell Dev Bi. 2017;33:319-342.

10. Nettersheim D, Berger D, Jostes S, Kristiansen G, Lochnit G, Schorle H. N6-Methyladenosine detected in RNA of testicular germ cell tumors is controlled by METTL3, ALKBH5, YTHDC1/F1/F2, and HNRNPC as writers, erasers, and readers. Andrology-Us. 2019.

11. Wang X, Lu Z, Gomez A, et al. N6-methyladenosine-dependent regulation of messenger RNA stability. Nature. 2014;505:117-120.

12. Shriwas $\mathrm{O}$, Mohapatra P, Mohanty S, Dash R. The Impact of m6A RNA Modification in Therapy Resistance of Cancer: Implication in Chemotherapy, Radiotherapy, and Immunotherapy. Front Oncol. 2020;10:612337.

13. Wang $X$, Wu R, Liu Y, et al. m(6)A mRNA methylation controls autophagy and adipogenesis by targeting Atg5 and Atg7. Autophagy. 2020;16:1221-1235.

14. Liu S, Li G, Li Q, et al. The roles and mechanisms of YTH domain-containing proteins in cancer development and progression. Am J Cancer Res. 2020;10:1068-1084.

15. Wang J, Wang J, Gu Q, et al. The biological function of m6A demethylase ALKBH5 and its role in human disease. Cancer Cell Int. 2020;20:347.

16. Song $H$, Feng $X$, Zhang $H$, et al. METTL3 and ALKBH5 oppositely regulate $m(6) A$ modification of TFEB mRNA, which dictates the fate of hypoxia/reoxygenation-treated cardiomyocytes. Autophagy. 2019;15:1419-1437.

17. Tang $B$, Yang $Y$, Kang $M$, et al. $m(6) A$ demethylase ALKBH5 inhibits pancreatic cancer tumorigenesis by decreasing WIF-1 RNA methylation and mediating Wnt signaling. Mol Cancer. 2020;19:3.

18. Zhang C, Samanta D, Lu H, et al. Hypoxia induces the breast cancer stem cell phenotype by HIFdependent and ALKBH5-mediated m6 A-demethylation of NANOG mRNA. Proceedings of the 
National Academy of Sciences. 2016;113:E2047-E2056.

19. Shriwas O, Priyadarshini M, Samal SK, et al. DDX3 modulates cisplatin resistance in OSCC through ALKBH5-mediated m(6)A-demethylation of FOXM1 and NANOG. Apoptosis. 2020.

20. Zhang J, Guo S, Piao HY, et al. ALKBH5 promotes invasion and metastasis of gastric cancer by decreasing methylation of the IncRNA NEAT1. J Physiol Biochem. 2019;75:379-389.

21. Yang P, Wang Q, Liu A, Zhu J, Feng J. ALKBH5 Holds Prognostic Values and Inhibits the Metastasis of Colon Cancer. Pathol Oncol Res. 2020;26:1615-1623.

22. Yang WS, SriRamaratnam R, Welsch ME, et al. Regulation of ferroptotic cancer cell death by GPX4. Cell. 2014;156:317-331.

23. Cao JY, Dixon SJ. Mechanisms of ferroptosis. Cell Mol Life Sci. 2016;73:2195-2209.

24. Imai H, Matsuoka M, Kumagai T, Sakamoto T, Koumura T. Lipid Peroxidation-Dependent Cell Death Regulated by GPx4 and Ferroptosis. Curr Top Microbiol Immunol. 2017;403:143-170.

25. Latunde-Dada GO. Ferroptosis: Role of lipid peroxidation, iron and ferritinophagy. Biochim Biophys Acta. 2017;1861:1893-1900.

26. Xie Y, Hou W, Song X, et al. Ferroptosis: process and function. Cell Death Differ. 2016;23:369-379.

27. Gorrini C, Harris IS, Mak TW. Modulation of oxidative stress as an anticancer strategy. Nat Rev Drug Discov. 2013;12:931-947.

28. Kumari S, Badana AK, G MM, G S, Malla R. Reactive Oxygen Species: A Key Constituent in Cancer Survival. Biomark Insights. 2018;13:91914689.

29. Madden SK, Itzhaki LS. Structural and mechanistic insights into the Keap1-Nrf2 system as a route to drug discovery. Biochim Biophys Acta Proteins Proteom. 2020;1868:140405.

30. Turpaev KT. Keap1-Nrf2 signaling pathway: mechanisms of regulation and role in protection of cells against toxicity caused by xenobiotics and electrophiles. Biochemistry (Mosc). 2013;78:111-126.

31. Salazar M, Rojo Al, Velasco D, de Sagarra RM, Cuadrado A. Glycogen synthase kinase-3beta inhibits the xenobiotic and antioxidant cell response by direct phosphorylation and nuclear exclusion of the transcription factor Nrf2. J Biol Chem. 2006;281:14841-14851.

32. Kim EH, Jang H, Shin D, Baek SH, Roh JL. Targeting Nrf2 with wogonin overcomes cisplatin resistance in head and neck cancer. Apoptosis. 2016;21:1265-1278.

33. Roh JL, Kim EH, Jang H, Shin D. Nrf2 inhibition reverses the resistance of cisplatin-resistant head and neck cancer cells to artesunate-induced ferroptosis. Redox Biol. 2017;11:254-262.

34. Shin D, Kim EH, Lee J, Roh JL. Nrf2 inhibition reverses resistance to GPX4 inhibitor-induced ferroptosis in head and neck cancer. Free Radic Biol Med. 2018;129:454-462.

35. Huang $\mathrm{H}$, Weng $\mathrm{H}$, Sun $\mathrm{W}$, et al. Recognition of RNA N(6)-methyladenosine by IGF2BP proteins enhances mRNA stability and translation. Nat Cell Biol. 2018;20:285-295.

36. Tang Z, Li C, Kang B, Gao G, Li C, Zhang Z. GEPIA: a web server for cancer and normal gene expression profiling and interactive analyses. Nucleic Acids Res. 2017;45:W98-W102.

37. Saito H. METABOLISM OF IRON STORES. Nagoya J Med Sci. 2014;76:235-254. 
38. Dodson M, Castro-Portuguez R, Zhang DD. NRF2 plays a critical role in mitigating lipid peroxidation and ferroptosis. Redox Biol. 2019;23:101107.

39. Lin H, Qiao Y, Yang H, et al. Small molecular Nrf2 inhibitors as chemosensitizers for cancer therapy. Future Med Chem. 2020;12:243-267.

40. Panieri E, Saso L. Potential Applications of NRF2 Inhibitors in Cancer Therapy. Oxid Med Cell Longev. 2019;2019:8592348.

41. Tang Z, Zhao L, Yang Z, et al. Mechanisms of oxidative stress, apoptosis, and autophagy involved in graphene oxide nanomaterial anti-osteosarcoma effect. Int J Nanomedicine. 2018;13:2907-2919.

42. Singh A, Venkannagari S, Oh KH, et al. Small Molecule Inhibitor of NRF2 Selectively Intervenes Therapeutic Resistance in KEAP1-Deficient NSCLC Tumors. Acs Chem Biol. 2016;11:3214-3225.

43. He L, Li H, Wu A, Peng Y, Shu G, Yin G. Functions of N6-methyladenosine and its role in cancer. Mol Cancer. 2019;18:176.

44. Panneerdoss S, Eedunuri VK, Yadav P, et al. Cross-talk among writers, readers, and erasers of $\mathrm{m}(6) \mathrm{A}$ regulates cancer growth and progression. Sci Adv. 2018;4:r8263.

45. Zhu H, Gan X, Jiang X, Diao S, Wu H, Hu J. ALKBH5 inhibited autophagy of epithelial ovarian cancer through miR-7 and BCL-2. J Exp Clin Cancer Res. 2019;38:163.

46. Xu T, Ding W, Ji X, et al. Molecular mechanisms of ferroptosis and its role in cancer therapy. J Cell Mol Med. 2019;23:4900-4912.

47. Fan Z, Wirth AK, Chen D, et al. Nrf2-Keap1 pathway promotes cell proliferation and diminishes ferroptosis. Oncogenesis. 2017;6:e371.

48. Agyeman AS, Chaerkady R, Shaw PG, et al. Transcriptomic and proteomic profiling of KEAP1 disrupted and sulforaphane-treated human breast epithelial cells reveals common expression profiles. Breast Cancer Res Treat. 2012;132:175-187.

49. Osburn WO, Wakabayashi N, Misra $\mathrm{V}$, et al. Nrf2 regulates an adaptive response protecting against oxidative damage following diquat-mediated formation of superoxide anion. Arch Biochem Biophys. 2006;454:7-15.

50. Ye J, Wang Z, Chen X, et al. YTHDF1-enhanced iron metabolism depends on TFRC m(6)A methylation. Theranostics. 2020;10:12072-12089.

51. Wang J, Wang J, Gu Q, et al. The biological function of m6A demethylase ALKBH5 and its role in human disease. Cancer Cell Int. 2020;20:347.

\section{Figures}


a

b
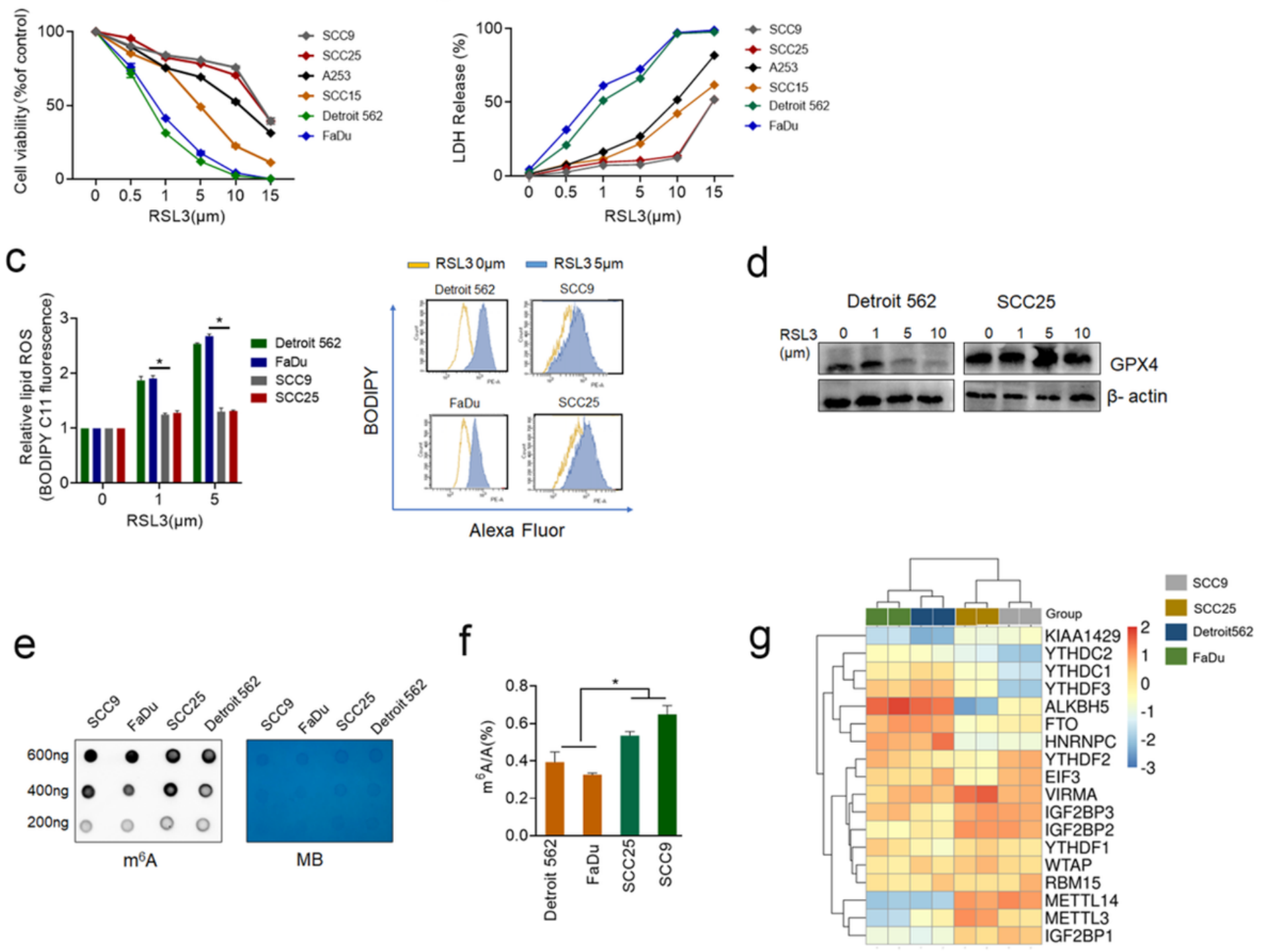

Figure 1

RSL3 induced head and neck cancer cell ferroptosis is closely correlated with global m6A abundance. a. b. Cell viability (a)and LDH release assays(b) of HNSCC cell lines that had been exposed to different concentrations of RSL3 for 24h. c. Measurement of cellular lipid ROS levels by using BODIPY C11 after exposure to RSL3 for $24 \mathrm{~h}$. d. Western blotting of Detroit 562 and SCC25 cells exposed to various concentrations of RSL3 for $24 \mathrm{~h}$. $\beta$-actin was used as a loading control. e. f. Representative images of m6A dot blot assay (e) and measurement of m6A quantitation (f) showed global m6A abundance in HNSCC cells. MB, methylene blue staining (as a loading control). g. Heat map of HNSCC cells with hierarchical clustering of ferroptosis-sensitive and ferroptosis-nonsensitive, each was normalized to the corresponding mean value. 

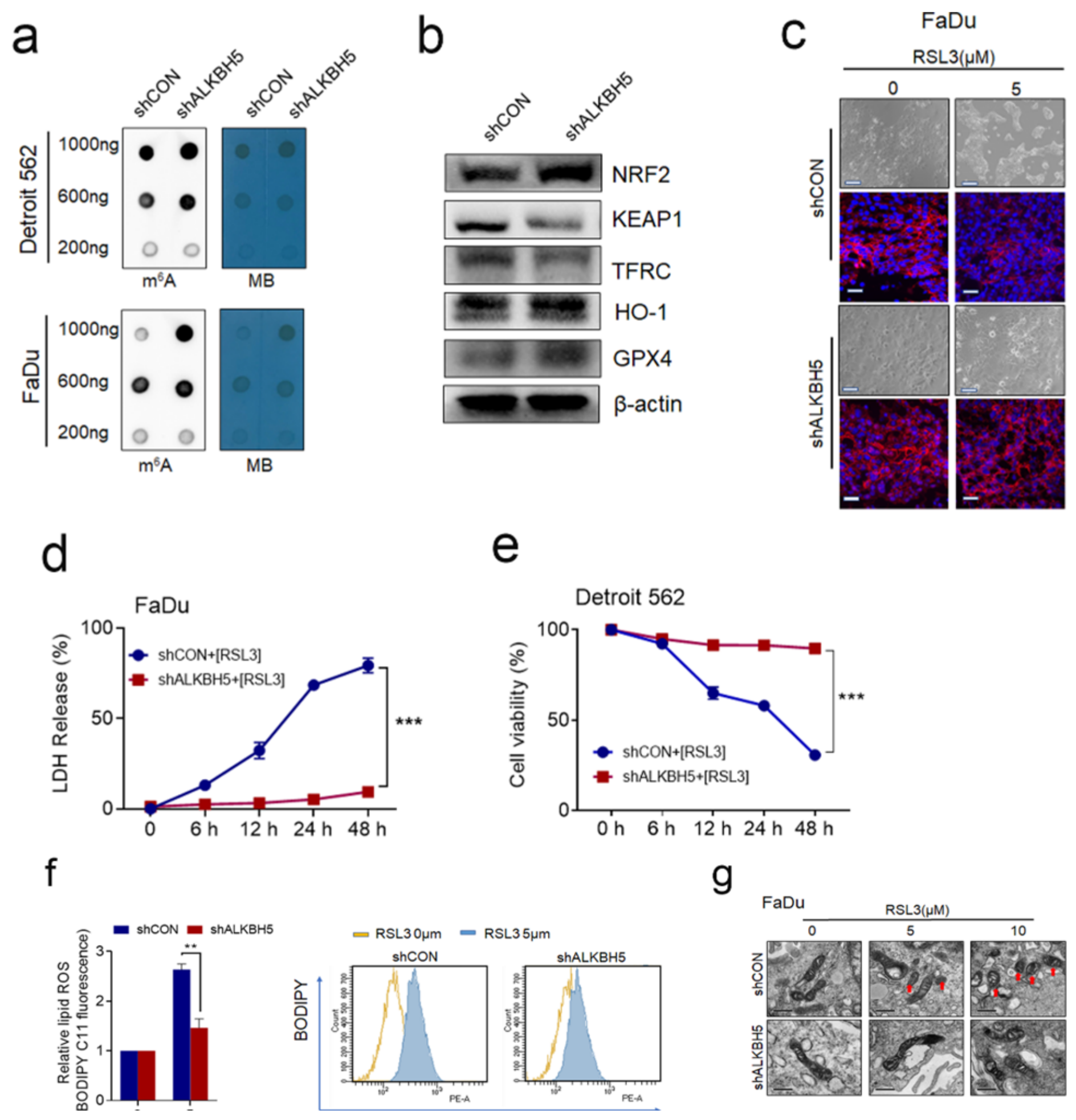

\section{Figure 2}

ALKBH5 drives to ferroptosis in HPSCC cells. a. The m6A dot blot assay of global m6A abundance in mRNA of control and ALKBH5-knockdown HPSCC cells. MB, methylene blue staining (as a loading control). b. Western blotting of indicated ferroptosis-related proteins in control and ALKBH5-knockdown Detroit 562 cells. $\beta$-actin was used as a loading control. c. Representative images of microscopy (Scale bar $=200 \mu \mathrm{m}$, Magnification: E, 20x.) and immunofluorescent staining for GPX4 (Scale bar=200 $\mu \mathrm{m}$, 
Magnification: E, 20x.) in control and ALKBH5-knockdown Detroit 562 cells 24h after RSL3 treatment patients. d. Cell viability assay of control and ALKBH5-knockdown Detroit 562 cells $24 \mathrm{~h}$ after RSL3 treatment. (Scale bar=200 $\mu \mathrm{m}$ Magnification: E, 20X) e. LDH release assay of control and ALKBH5knockdown Detroit 562 cells 24h after RSL3 treatment. f. Measurement of cellular lipid ROS levels in control and ALKBH5-knockdown Detroit 562 cells by using BODIPY C11 after exposure to RSL3 $(5 \mu \mathrm{m})$ for $24 \mathrm{~h}$. g. Ultrastructural analysis of shCON and shALKBH5 cells. Red arrows indicate outer membrane rupture (scale bars, $500 \mathrm{~nm}$ ).
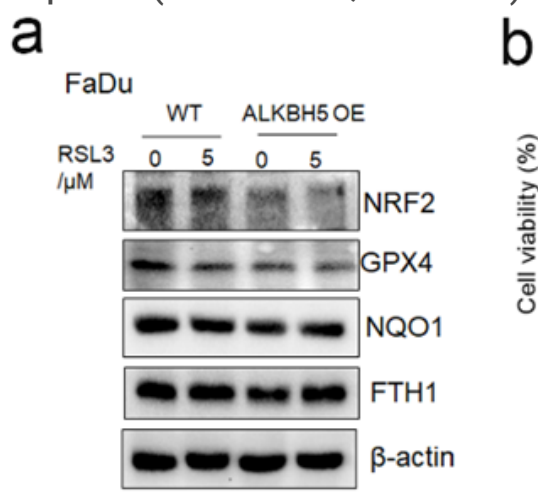

d

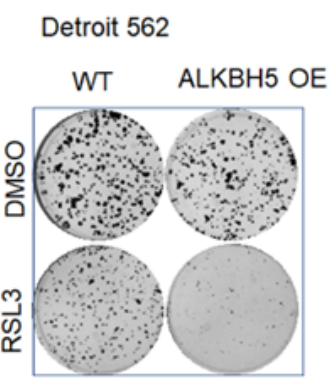

b

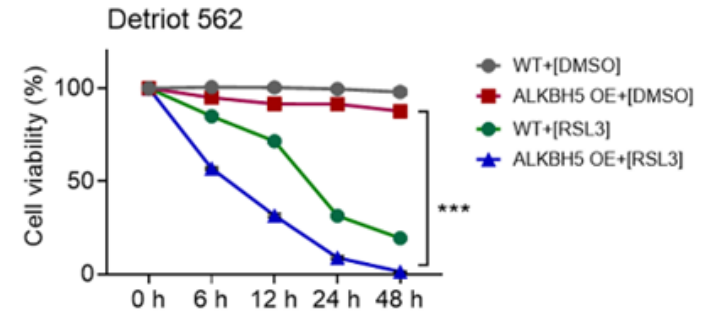

e

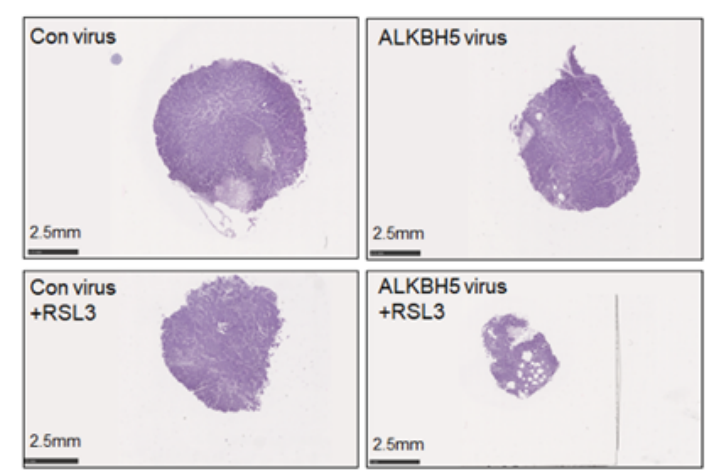

C

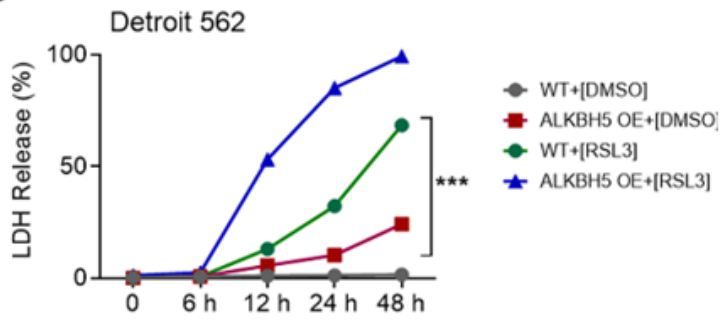

f
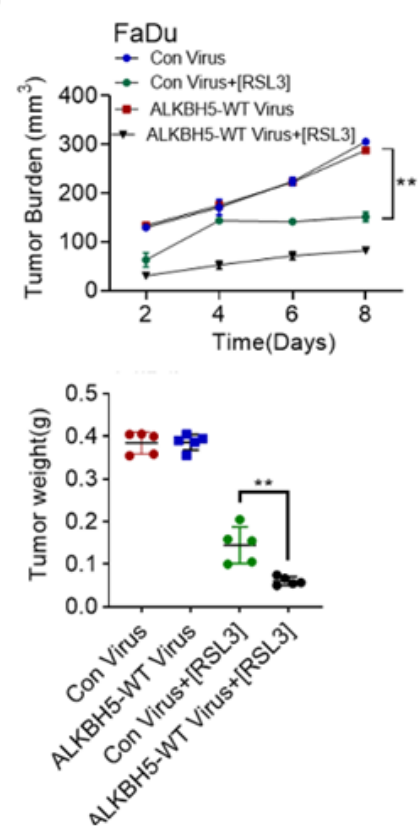

\section{Figure 3}

The overexpression of ALKBH5 sensitized HPSCC cells to RSL3 treatment in vitro and vivo a. b. c. Cell viability assay (a), LDH release assay(b) and colony formation assays (c) were performed with FaDu cells transfected with a control vector or ALKBH5-WT virus and subsequently treated with $5 \mu \mathrm{m}$ RSL3. d. e. f. Representative images of H\&E-stained tissues to evaluate xenograft tumor formation (d), tumor volumes (e), and weight (f) in nude mice bearing Detroit 562 cells transfected with a control vector or ALKBH5-WT virus with or without RSL3 treatment. 
a

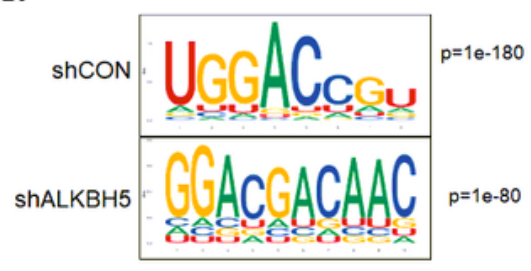

b

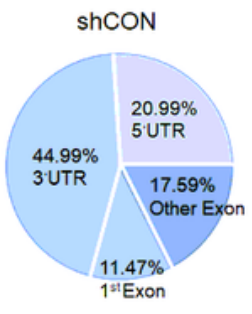

C

Fig.4

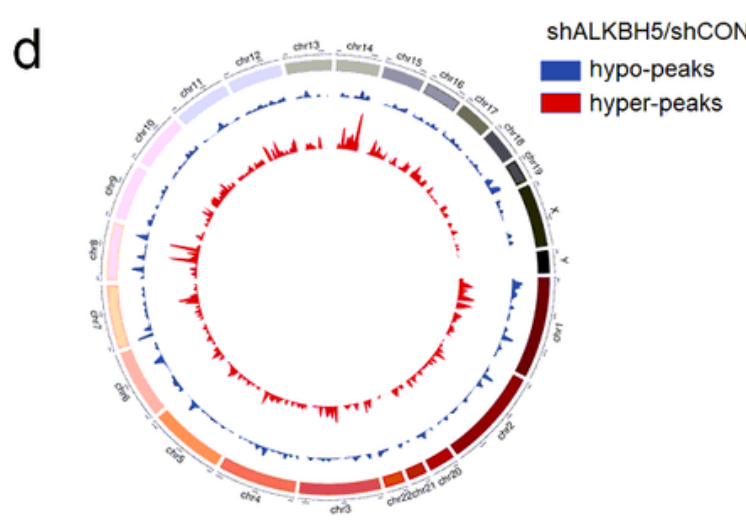

f

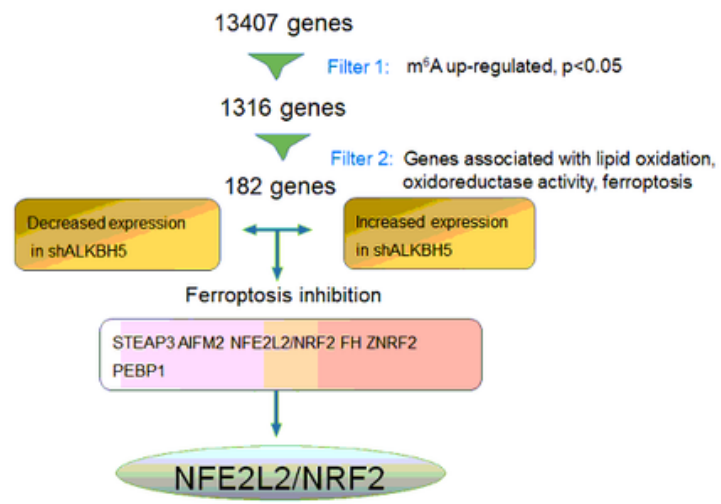

i g

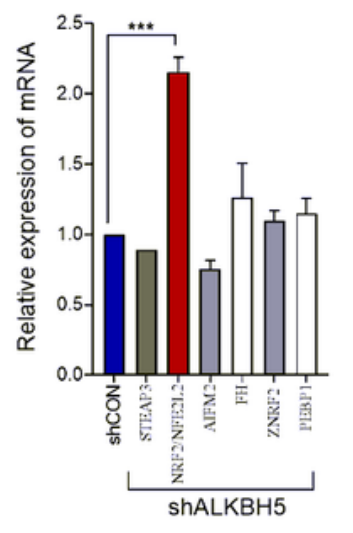

NFE2L2/NRF2

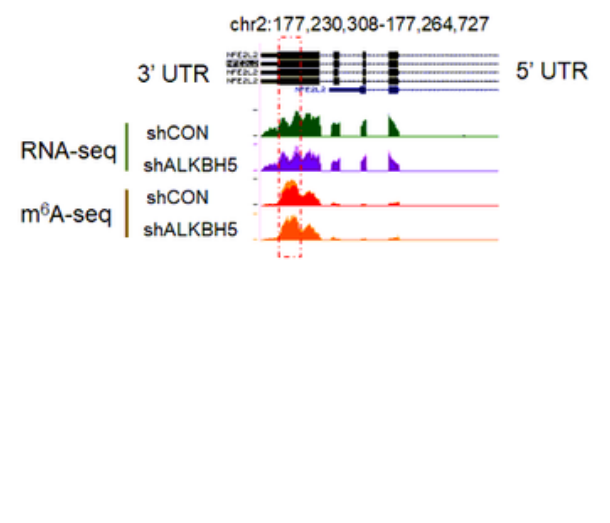

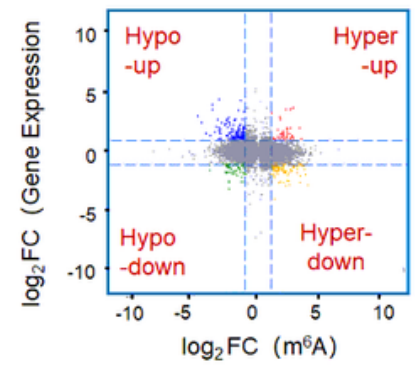

h
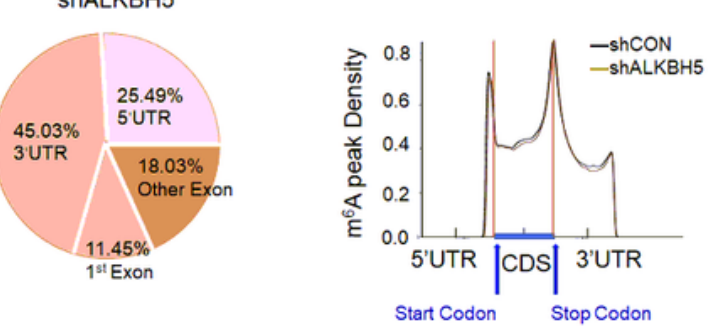
untranslated region (3'UTR) were divided into 100 segments, and the percentages of peaks within each segment weredetermined. c. Proportion of m6A peak distribution in the 5'UTR, start codon, CDS, stop codon and 5'UTR region in the entire set of mRNAtranscripts. d. Circos plot showing the distribution of hypermethylated m6A (hyper) and hypomethylated (hypo) peaks in the human transcriptome of FaDu shALKBH5 cells compared with shCON cells. e. Distribution of genes with a significant change in both m6A level (log2FC) and gene expression level (log2 FC) in the shCON and shALKBH5 FaDucells. $f$.

Relative RNA level of indicated gene in FaDu cells upon ALKBH5 knockdown. g. Flow chart of the selected candidate ALKBH5 target genes in FaDu cells. h. IGV tracks displaying the distribution of m6A peaks and ALKBH5-binding peaks among the indicated genes according to m6A-seq and RNA-seq of FaDu cells. i. Sequence analysis of NFE2L2/NRF25'-UTR revealed two matches to the 5'-RRACA-3' (R=G or A) m6A consensus sequence. 


\section{Fig.5}
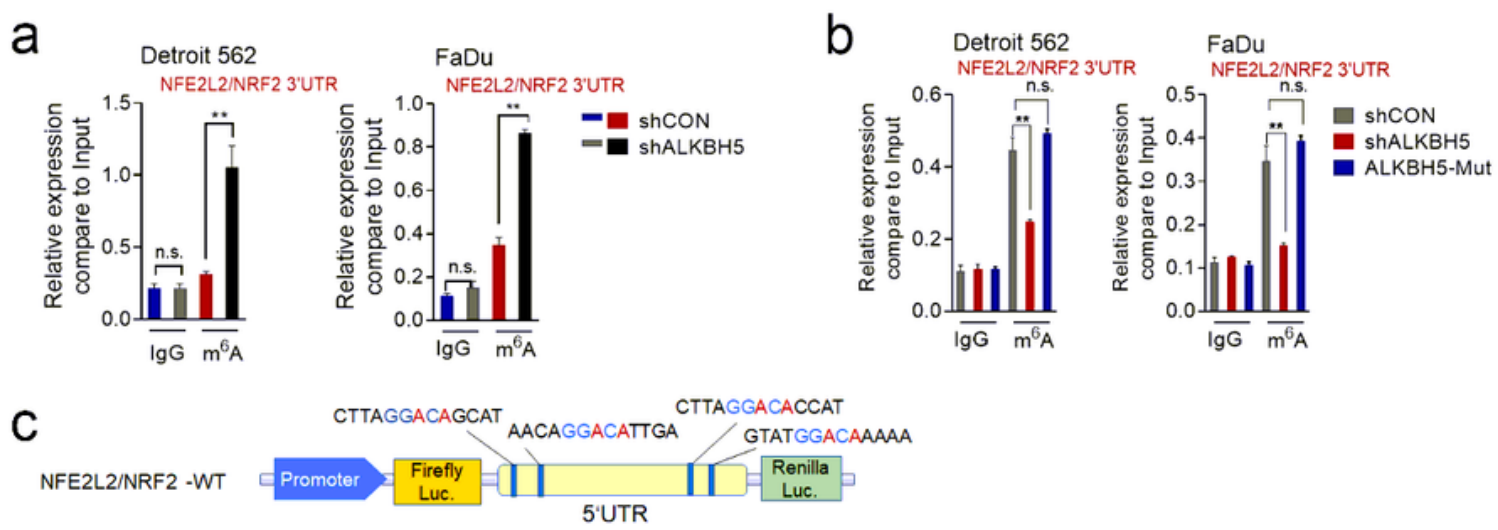

NFE2L2/NRF2 -Mut CtTAGgAcagCAT AaCAGGACATTGA CTTAGGACACCAT
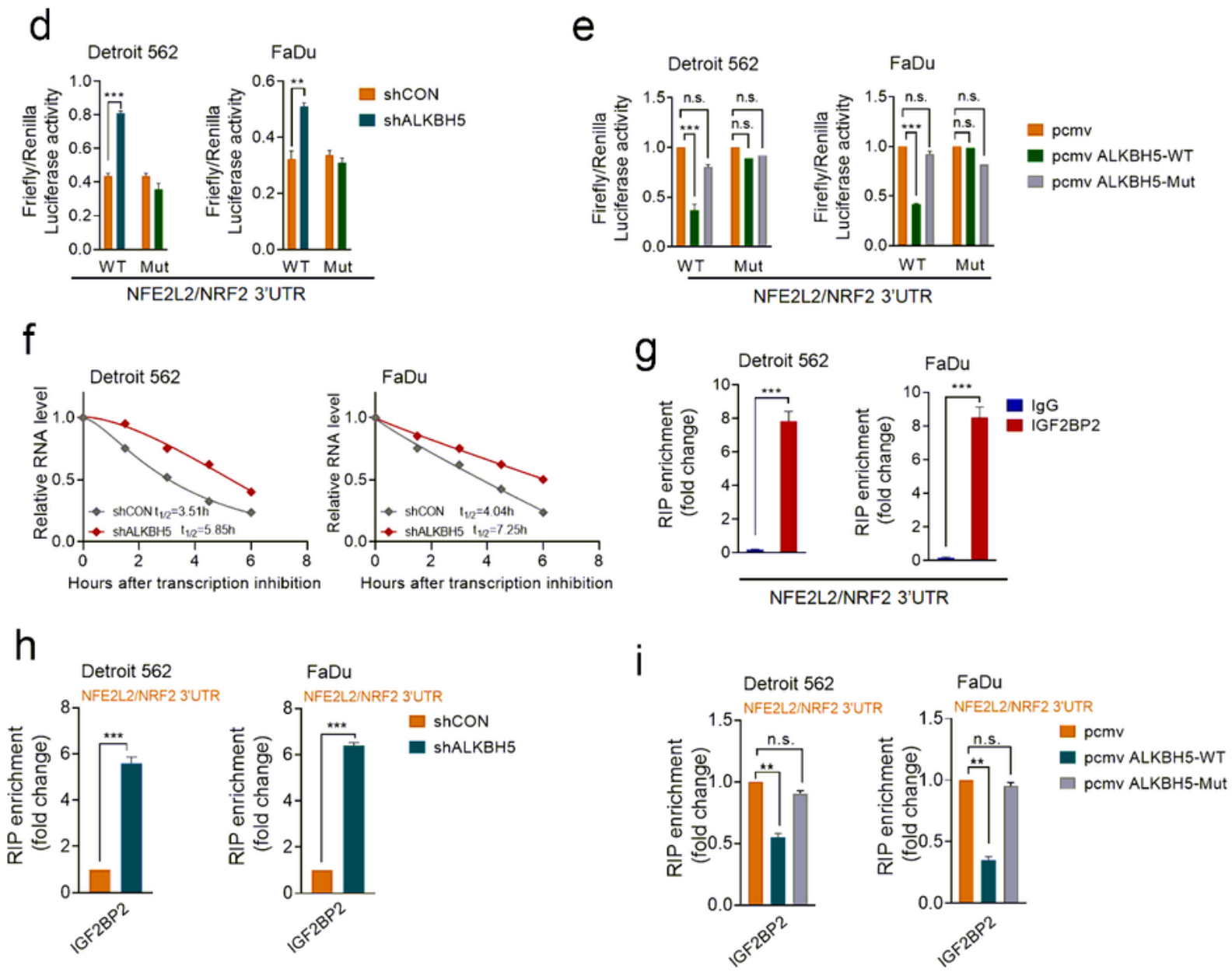

\section{Figure 5}

ALKBH5 abolishes NFE2L2/NRF2mRNA levels and stability depending on m6A methyltransferase activity. a. MeRIP-qPCR analysis of NFE2L2/NRF2 3'UTR m6A levels in control and ALKBH5-knockdown Detroit 562 and FaDu cells, $n=3$, nonparametric Mann-Whitney test. b. MeRIP-qPCR analysis of NFE2L2/NRF2 3'UTR m6A levels GV141, GV141-ALKBH5-mut (H204A mutant of ALKBH5) and GV141ALKBH5-WT Detroit 562 and FaDu cells, $n=3$, nonparametric Mann-Whitney test. c. Graphical 
explanation for construction of luciferase reporters expressing WT or mutant human NFE2L2/NRF23'UTR s. Relative luciferase activity was computed by the ratio of Firefly and Renilla luciferase values. $d$. e. The luciferase reporters expressing WT or mutant human NFE2L2/NRF2-3'UTRs. Luciferase activity was measured in Detroit 562 and FaDu cells transfected with control shCON and shALKBH5 (d), and GV141, GV141-ALKBH5-mut (H204A mutant of ALKBH5) and GV141-ALKBH5-WT (e), n = 3, nonparametric Mann-Whitney test. $f$. The NFE2L2/NRF2 mRNA half-life (t 1/2) was detected by real-time PCR in control and ALKBH5-knockdown Detroit 562 and FaDu cells. Cells were treated with actinomycin D and harvested at indicated times. $n=3$, nonparametric Mann-Whitney test. g. RIP-qPCR analysis in HPSCC cells showing the direct binding between the IGF2BP2 protein with NFE2L2/NRF2-3'UTRs. $\mathrm{h}$. i. RIP-qPCR analysis revealed the binding enrichment of IGF2BP2 to NFE2L2/NRF2-3'UTRs in HPSCC cells transfected with control shCON and shALKBH5 (h), and GV141, GV141-ALKBH5-mut (H204A mutant of ALKBH5) and pcmv-ALKBH5-WT (i), $n=3$, nonparametric Mann-Whitney test. 
a

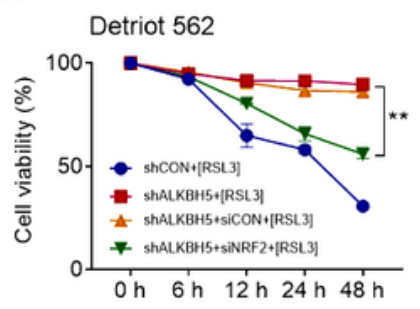

b

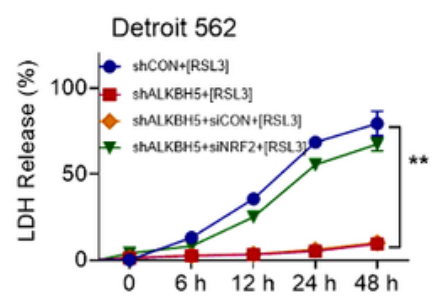

Fig.6

C

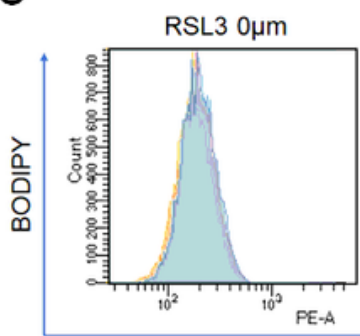

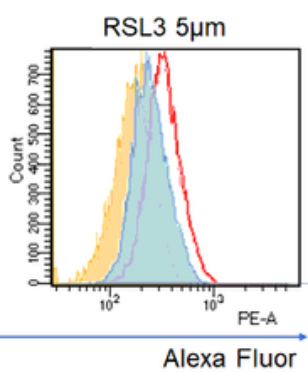

- shCON

$\square$ shALKBH5+siCON

- shALKBH5+siNRF2

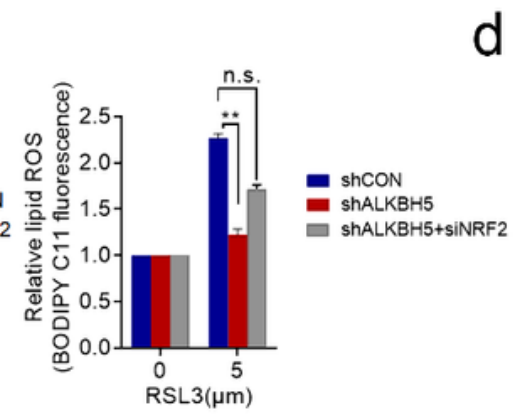

Detroit 562

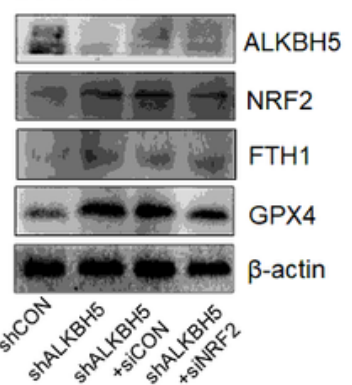

e

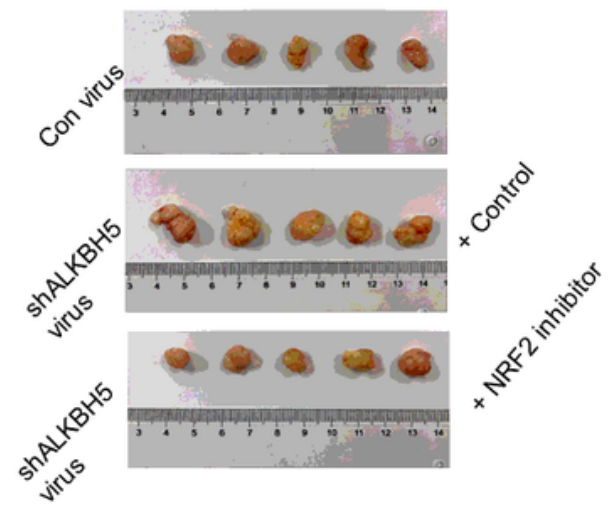

f

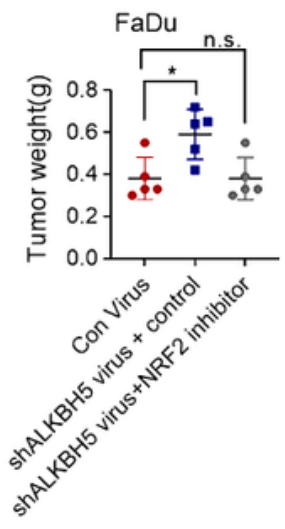

g

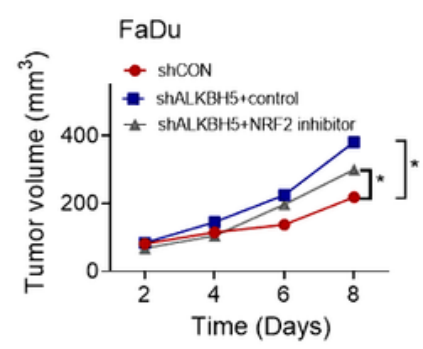

h

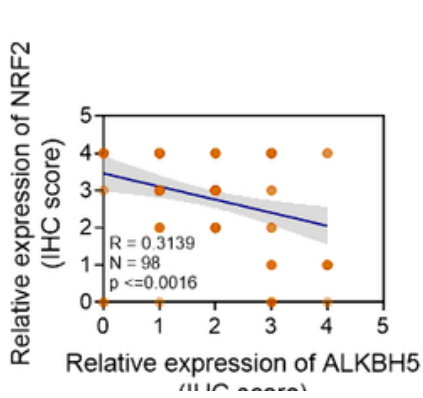

i

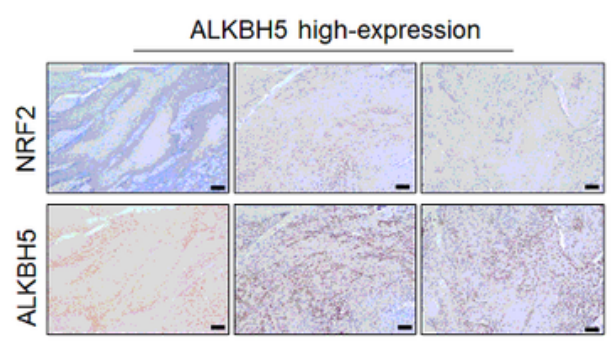

ALKBH5 low-expression

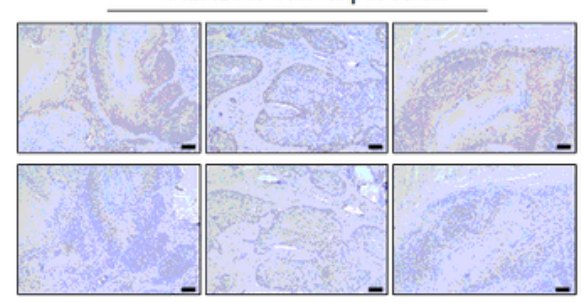

\section{Figure 6}

NFE2L2/NRF2 is functionally essential target gene of ALKBH5 in HPSCC. a. b. Cell viability assay (a), LDH release assay (b) were performed with control and shALKBH5 Detroit 562 cells transfected with a control vector (siCON) or NFE2L2/NRF2 siRNA (siNRF2) and subsequently treated with $5 \mu \mathrm{m}$ RSL3. c. Western blotting assays were performed to analyze NFE2L2/NRF2,GPX4,FTH1 levels after silencing NFE2L2/NRF2 (siNRF2) in Detroit 562 control and shALKBH5 cells. d. Measurement of cellular lipid ROS 
levels after transfection with siCON or siNRF2 in control and ALKBH5-knockdown Detroit 562 cells by using BODIPY C11 after exposure to RSL3 $(5 \mu \mathrm{m})$ for $24 \mathrm{~h}$. e. f. g. Representative images of H\&E-stained tissues to evaluate xenograft tumor formation (e), tumor weight (f) and volumes ( $g$ ) in nude mice bearing FaDu cells transfected with a control vector or shALKBH5 virus with or without ML385 treatment (30 mg $/ \mathrm{kg}$, intraperitoneal injection). $\mathrm{X}$ Q $\mathrm{h}$. Pearson's rank correlation of ALKBH5 and NFE2L2/NRF2 proteins in HPSCC tissues from patients based on the IHCanalysis. i. Representative IHC images of ALKBH5 and in NFE2L2/NRF2 HPSCC tissues from patients. Scale bar $=100 \mu \mathrm{m}(40 \times)$

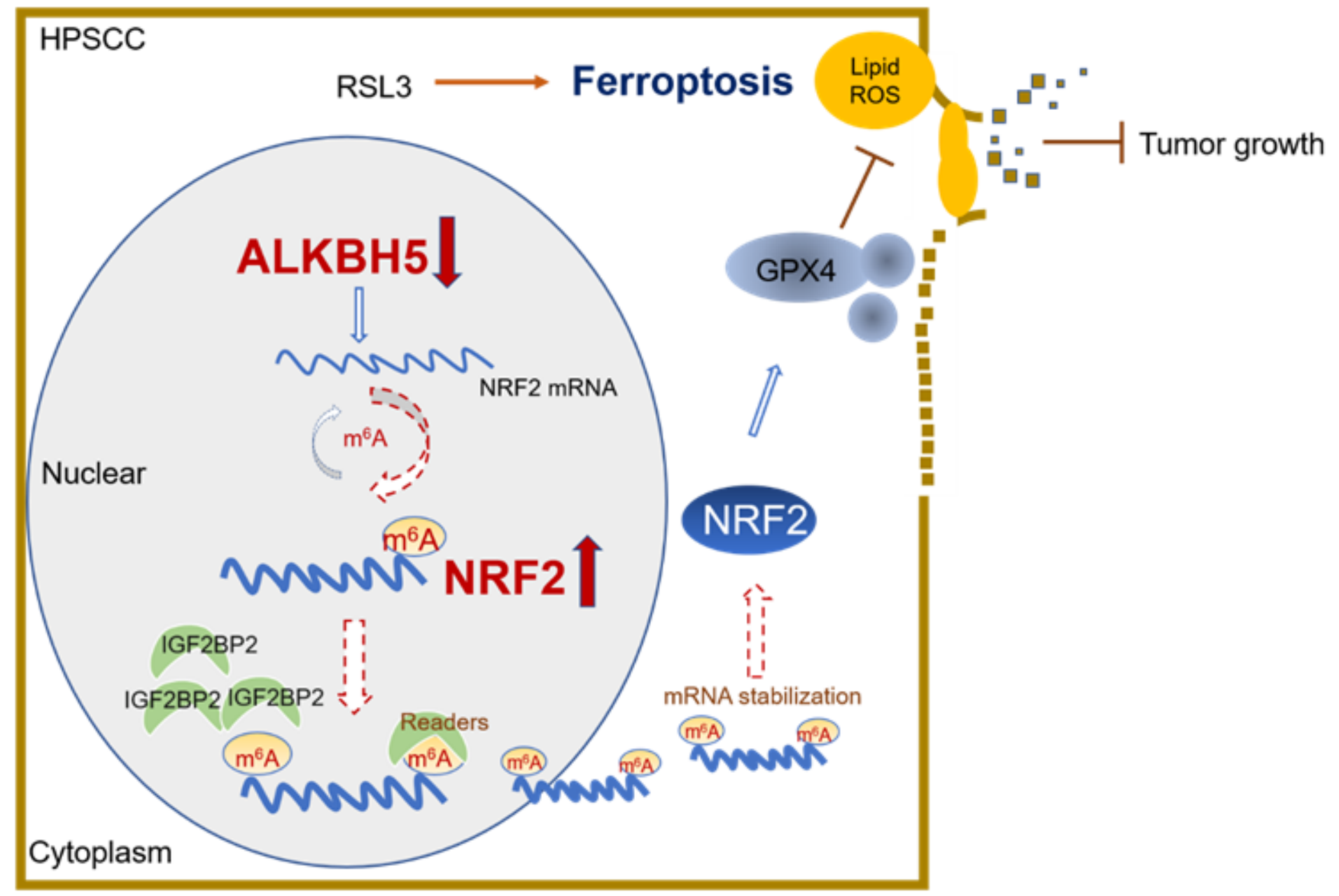

\section{Figure 7}

A schematic illustration was proposed to summarize the relationship among ALKBH5,m6A modification, cell death and ferroptosis.

\section{Supplementary Files}

This is a list of supplementary files associated with this preprint. Click to download.

- Supplementaryfigures.docx

- Supplementary3.xls 
- supplementary1.docx

- supplementary2.docx 